\title{
Cooperação multilateral em saúde: a Oitava Conferência Internacional sobre Promoção da Saúde
}

\author{
Multilateral cooperation on health: the Eight International Conference on Health \\ Promotion
}

\section{Douglas Marcos Pereira de Paula}

Graduando em Gestão de Serviços de Saúde, Extensionista, Universidade Federal de Minas Gerais, Belo Horizonte, Brasil.

\section{Mateus Aparecido de Faria}

Graduando em Gestão de Serviços de Saúde, Extensionista, Universidade Federal de Minas Gerais, Belo Horizonte, Brasil.

\section{Jullien Dábini Lacerda de Almeida}

Graduando em Gestão de Serviços de Saúde, Extensionista, Universidade Federal de Minas Gerais, Belo Horizonte, Brasil.

Resumo: A cooperação internacional pode acontecer de diferentes formas: bilateral, multilateral ou ainda entre países em desenvolvimento, como exemplo a cooperação Sul-Sul. Entre as diversas formas destacam-se algumas instituições e organismos como a Organização Mundial da Saúde (OMS), a Organização das Nações Unidas e a Organização Pan-Americana da Saúde. A OMS, se utilizando de instrumentos para cooperação multilateral como as conferências globais sobre promoção da saúde, vem complementar esforços a fim de contribuir para a cooperação internacional em saúde ascendendo a cidadania dos povos. Descreve-se a Oitava Conferência Internacional sobre Promoção da Saúde cujo tema foi 'saúde em todas as políticas e suas nuances nas prerrogativas no campo da saúde'; a influência dos países presentes na elaboração da declaração final e analisam-se as declarações paralelas. Sediada na cidade finlandesa de Helsinki entre os dias 10 e 14 de junho de 2013, a Conferencia marca a importância das políticas públicas e sua interferência nos determinantes da saúde e bem-estar, resgatando os preceitos ideários da Declaração de Alma-Ata e da Carta de Ottawa. A Declaração de Helsinki defende, entre outros princípios, a intersetorialidade na saúde. Paralelamente, o movimento saúde dos povos também sublinha pontos propondo alternativas ao documento oficial.

Palavras-chaves: Cooperação internacional; cooperação técnica; promoção da saúde; Direito Internacional.

Key-words: International cooperation; technical cooperation; health promotion; International Law. 


\section{Introdução}

A cooperação internacional multilateral, definida como um ato solidário segundo a Agência Brasileira de Cooperação ( $A B C)$, ligada ao Ministério das Relações Exteriores brasileiro, vai de encontro à proposta subliminar - positivamente - das Conferências Internacionais sobre a Promoção da Saúde.

O termo cooperação internacional muitas vezes é entendido como sobreposição de países desenvolvidos a países em desenvolvimento referente ao tipo bilateral de acordo ou de uma cooperação multilateral solidária, como preconizado pela $A B C$. A conferências funcionam a partir da mescla desses tipos de cooperações uma vez que chama para o debate países desenvolvidos e em desenvolvimento (vistos a partir do prisma econômico) a fim de se deliberar e delinear sobre o cenário internacional. Desde a primeira conferência realizada em 1986, na cidade de Ottawa, Canadá, até a oitava, sediada em Helsinki, Finlândia, ambas promovidas pela Organização Mundial da Saúde (OMS), fomentam a necessidade de se discutir, horizontalmente, estratégias que visam garantir o direito e melhorias da saúde dos povos.

A primeira Conferência Internacional de Promoção da Saúde buscou um conjunto de princípios desencadeadores para ações de promoção de saúde como equidade, cidadania, participação, solidariedade, com o objetivo Saúde para Todos no ano 2000. A segunda Conferência, realizada em Adelaide, Austrália, em 1988, destacou quatro áreas emergenciais de âmbito político: tabaco e álcool, apoio a saúde da mulher, alimentação e nutrição e criação de ambientes favoráveis à saúde. Após três anos, em Sundsvall, Suécia, a terceira edição da conferência resgatou aspectos da Declaração de Adelaide, além de ligar ecologia e saúde, e destacava as dimensões sociais, econômicas e políticas como necessárias para a promoção da saúde nos anos 1990. A inovação nas conferências se deu em 1997: Indonésia, o primeiro país em desenvolvimento a sediar uma conferência internacional para discussão sobre como promover saúde, incluindo o setor privado nos debates da Conferência.

A partir do insucesso do lema Saúde para Todos no ano 2000, a quinta Conferência, sediada na capital do México, trouxe à tona o papel da participação popular para consolidação da saúde a nível local e a responsabilização dos diferentes níveis de poder pela saúde de sua comunidade. Com um jejum de cinco anos e agora 
com sede em Bangkok, Tailândia, a sexta Conferência introduziu o mundo globalizado no tema - trazendo o envelhecimento populacional, crescimento de doenças crônicas e transmissíveis, rápida urbanização e degradação do meio ambiente. Assumiu-se, aqui, a responsabilização de novas ações de promoção da saúde a partir de quatro diretrizes: responsabilidade governamental, desenvolvimento de agendas globais, necessidade de boa gestão da pratica e priorização dos anseios da sociedade civil. Em 2009, a sétima edição da Conferencia, realizada em Nairóbi, Quênia, discutiu os impasses para implementação inserindo nas agendas políticas e de desenvolvimento um mecanismo efetivo de distribuição de renda. Por fim, a oitava Conferência sobre Promoção da Saúde: Saúde em Todas as Políticas, realizada em Helsinki, Finlândia, entre dias 10 e 14 de junho de 2013.

É importante salientar que devido a algumas conferências internacionais terem acontecido em espaços irregulares de tempo, houve encontros importantes sobre questões ligadas à promoção da saúde: 1) em 1992, Declaração de Santa-fé de Bogotá; 2) em 1993, Primeira Conferencia de Promoção da Saúde do Caribe, Caribe; 3) em 1996, Promoção à Saúde da População no Canadá; 4) em 1998, Rede de Mega Países para a Promoção da Saúde; 5) em 2011, Declaração Política do Rio sobre Determinantes Sociais da Saúde; 6) e Quinta Conferência Latino-americana de Promoção da Saúde e Educação para a Saúde.

Outro marco importante que deve ser destacado é a Declaração do Milênio das Nações Unidas no ano de 2000, em Nova York, onde os lideres defiram metas como eliminar a fome e a pobreza no mundo: educação básica de qualidade para todos, igualdade entre os sexos, redução da mortalidade infantil, melhoria da saúde materna, combate a doenças epidêmicas como AIDS e malária, garantia da sustentabilidade ambiental e estabelecimento de cooperações internacionais para o desenvolvimento.

Portanto, a questão norteadora que guiou esse estudo foi: como foi a oitava Conferência Global de Promoção da Saúde e quais foram os resultados obtidos?

\section{Metodologia}

O artigo busca descrever a Oitava Conferencia Global sobre Promoção da Saúde, cujo tema é 'Saúde em todas as Políticas', utilizando uma abordagem qualitativa descritiva conceitual e analisar as cartas assinadas ao final do evento. 
As fontes utilizadas foram o site oficial do evento, onde se encontravam as experiências descritas e as cartas paralelas, lançadas oficialmente por suas respectivas entidades.

\section{Resultados}

\section{A Conferência: contextualização}

A Finlândia é um país nórdico da Europa, cuja capital é Helsinki. De clima temperado continental, sua população gira em torno de cinco milhões de habitantes. O país foi escolhido devido ao cumprimento do "dever de casa" quanto à promoção da saúde: baixa mortalidade infantil em relação ao resto da Europa, elevada expectativa de vida (em torno de 80 anos) e 100\% da população alfabetizada.

Com foco nos meios de promover saúde e equidade, pela conjugação de todos os setores governamentais, essa Conferência buscou a importância das políticas públicas e sua importância nos determinantes da saúde e bem-estar. Realizada em cinco dias, chefes de Estado discutiram os avanços de Ottawa até Helsinki e os processos de implementação da saúde em todas as políticas; as questões econômicas que interferem no processo e os desafios e oportunidades em todas as políticas em termos de dificuldade.

Durante a programação da conferência, entre plenárias e sessões, os chefes de Estado, representantes do Banco Mundial e de outras organizações ligadas à saúde dos povos apresentaram experiências exitosas sobre promoção da saúde.

O Brasil apresentou uma síntese do programa de transferência de renda direta - Bolsa Família - e o progresso dessa política de proteção social; Equador apresentou o Sistema Nacional de Inclusão e Equidade Social, mostrando a efetivação da intersetorialidade em seus processos constituintes; Turquia apresentou um Projeto de Redução do Uso do Tabaco por Adultos através de uma motivação de toda sociedade civil se apropriando de canais de mídia; Dinamarca apresentou seu Programa de Saúde e Seguridade Ocupacional, bem com suas metas para 2020; Estônia e Rússia apresentaram suas experiências na prevenção de doenças crônicas não transmissíveis; Holanda apresentou o Programa de Escolas Promotoras da Saúde; Suécia, em caráter de debate, apontou a revisão do conceito de pessoa idosa como recurso ativo e não como um peso; Alemanha trouxe lições de como é possível e factível políticas de redução da poluição do ar; El Salvador mostrou seu Sistema de 
Saúde Nacional e a luta da sociedade pela não privatização. Como resultados das discussões e das experiências compartilhadas, ao fim da conferência, países se reuniram em blocos com o intuito de elaborar os compromissos para afirmar a saúde em todas as políticas. A Carta oficial, de caráter internacional, aponta os caminhos definidos na Conferência bem como os compromissos dos governantes com a saúde.

\section{A Carta como acordo para a 'Saúde em Todas as Políticas'}

A Carta de Helsinque conclama à intersetorialidade na formação de políticas públicas e a necessidade de vontade política dos governantes para a efetivação da promoção de saúde dos povos. Seus signatários reconhecem que o acesso ao mais alto nível de gozo da saúde é um dos direitos fundamentais de todo ser humano, independente de gênero, credo, condição social e cultural ou quaisquer outras características que diferenciem os seres humanos. Além disso, afirma que a equidade é necessária para se ter uma sociedade justa e com maior qualidade de vida, sendo a iniquidade em saúde um atributo inaceitável em âmbito social, político e econômico, bem como evitável pelos governantes. A globalização, a rápida urbanização e as alterações demográficas também foram reconhecidas como fatores ativos, tanto para beneficiar quanto para atrapalhar as ações de promoção de saúde, sendo mais efetivos em países em desenvolvimento.

A ênfase no consumismo foi marcada pela sua influência nos estilo e modos de vida e em ambientes de trabalho, prejudicando-os. Faz-se necessária a vontade, coragem e disposição políticas para ampliar a saúde a outros setores governamentais e para desafiar interesses comerciais, de mercado e as forças poderosas econômicas, uma vez que estas criam obstáculos para que o Estado e a sociedade possam proteger e promover saúde em seus espaços.

Salientaram que o lema 'Saúde em Todas as Políticas' é parte integrante para o atingimento dos Objetivos de Desenvolvimento do Milênio, como também é essencial para a elaboração da Agenda de Desenvolvimento pós-2015.

Ficaram estabelecidos como objetivos: priorizar a saúde e a equidade como responsabilidade central dos governos; afirmar a necessidade de coerência política e reconhecer a necessidade de vontade política, coragem e visão estratégica. Estabeleceram-se, assim, obrigações para a OMS e os governos, responsabilizando ambos para o seu cumprimento com bem-estar e a saúde dos povos. 
A Carta termina com uma chamada para a ação: "Comprometemo-nos a comunicar as principais mensagens desta Declaração de Helsinki aos nossos governos, instituições e comunidades".

\section{Cartas paralelas}

Durante a oitava conferencia foram elaboradas declarações paralelas, provenientes da delegação El Salvador e do Movimento Saúde dos Povos, como o objetivo de criticar, fortalecer e apontar alternativas à decisão oficial.

\section{A carta de El Salvador}

Resgatando os pontos chave da Declaração Universal dos Direitos Humanos, das conferencias realizadas anteriormente, da Carta dos Povos pela Saúde de 2000, da Declaração de Cuenca de 2005, o Chamado para Ação de Cape Town e das reformas de seu Sistema Nacional de Saúde, a delegação de El Salvador propôs uma declaração paralela à Conferência.

Ela 1) denuncia as relações de consumo e comerciais desiguais que perpetuam e aprofundam as desigualdades internacionais, causando impactos internacionais principalmente em países em desenvolvimento; 2) denuncia a relação de exposição de agrotóxicos a doenças renais crônicas em populações agrícolas de países da America Central, Egito, Sirilanka, Vietnam e muitas outras regiões do mundo; 3) chama para responsabilização das causas e efeitos das mudanças climáticas os países industrializados, para assumirem medidas políticas e econômicas favoráveis ao meio ambiente mundial; 4) inclui a participação e organização da sociedade e dos governos para a defesa da vida; 5) incentiva a produção de conhecimento para o desenvolvimento dos sistemas nacionais de saúde; e 6) exige a participação popular para a formação de políticas sociais, considerando a estabilidade do ecossistema e colocando as necessidades das populações acima do econômico e dos interesses mercantis.

\section{A Carta do Movimento Saúde dos Povos}

Reunidos durante a oitava Conferência, ativistas do Movimento Saúde dos Povos elaboraram uma declaração paralela a fim de criticar a decisão oficial e desenvolver uma chamada para ação com base em princípios da justiça social. 
Assim, contextualiza a nova ordem econômica social apontando para 1) a crise do desenvolvimento sustentável, neoliberalismo e consumismo sobrepondo os valores da comunidade e solidariedade internacional; 2) a negação do direito a emprego decente, proteção social, segurança alimentar e demais determinantes sociais da saúde à centenas de milhões de pessoas; 3) a formação de conflitos e ações violentas enraizados em desigualdades e ganâncias corporativas; 4) a migração em grande escala para cidades e para alem das fronteiras internacionais; e 5) a desigualdades de renda e riqueza entre e dentro dos países.

Essa declaração também sublinha a urgência das ações exigidas pela OMS em conferências realizadas anteriormente e elogia a liderança da diretora geral da Organização, Margaret Chan, e sua posição contra as grandes indústrias e seus impactos destrutivos.

Reconhecendo que a Declaração de Helsinki não foi suficiente para indicar os caminhos nem as ações para se promover a saúde integralmente, o Movimento Saúde dos Povos estabelece dezessete pontos entre os quais estão: fortalecimento dos Estados-membros da OMS e seu papel de liderança na área da saúde; resolução de conflito pela OMS decorrente de contribuições orçamentarias voluntarias de corporações transnacionais, principalmente as farmacêuticas e militares; democratização da governança de entidades globais, incluindo a ONU, pelos Estados Unidos da América; adoção e avaliação da abordagem, por todos os níveis de governo, quando se trata de saúde em todas as políticas; implementação de impostos de renda progressivos, baseados na riqueza das pessoas físicas e jurídicas, a fim de controlar a especulação global e financiar ações sobre os determinantes sociais em saúde; segurança de que a saúde seja uma prioridade no comércio internacional; a incondicionalidade das doações e promoção da igualdade entre países de níveis diferentes de renda e alteração do regime de propriedade intelectual a fim de partilhar equitativamente conhecimentos, tecnologias e dados científicos tornando-os bens públicos globais.

\section{Considerações finais}

A partir desses resultados, entende-se que as Conferencias Internacionais sobre Promoção da Saúde têm, como dispositivo norteador, a intenção de colocar em pauta discussões globais sobre as políticas implementadas de promoção de saúde 
em diversos países e compartilhar com os demais as experiências exitosas e a possível solução para uma maior cidadania dos povos.

A cooperação multilateral se dá na reunião de vários países em torno de uma entidade internacional e é própria de sua natureza existir acordos ou cartas de intenção paralelas às firmadas oficialmente.

A Carta de El Salvado expressa sua indignação, como país considerado em desenvolvimento, às ações mercadológicas, não encontrando caminhos alternativos para o progresso de acordo com sua história e se alienando aos acordos abusivos do neocolonialismo. Já a Declaração do Movimento Saúde dos Povos exprime a centralização da responsabilidade na ONU e na OMS e suas agências, listando aspectos fundamentais na construção da Saúde em Todas as Políticas.

Não há informações suficientemente acerca do por que dos locais das Conferencias Internacionais sobre Promoção da Saúde - tenta-se deduzir as características buscando dados sobre aquela determinada população através dos relatórios emitidos pela OMS, pelos cenários históricos e aspectos demográficos.

Por fim, são necessárias mais pesquisas e a produção de conhecimento e sua divulgação em língua portuguesa na área de organismos internacionais de saúde e seus impactos nos sistemas nacionais de saúde por considerarmos que as cooperações multilaterais, apesar de aparentemente serem horizontais, escondem relações verticais prejudiciais para a igualdade dos países.

\section{Referências}

AGÊNCIA BRASILEIRA DE COOPERAÇÃO. Diretrizes para o Desenvolvimento da Cooperação Técnica Internacional Multilateral e Bilateral. (2. ed.) Brasília, 2005. [consultado em 10 ago. 2013] Disponível em: http://www.abc.gov.br/Content/ABC/docs/DiretrizesCTI new2.pdf

AZEVEDO, Elaine de; PELICIONI, Maria Cecília Focesi. Promoção da Saúde, Sustentabilidade e Agroecologia: uma discussão intersetorial. Saude soc. [online]. 20(3):715-729, 2011. [consultado em 13 jun. 2013] Disponível em: http://www.scielo.br/scielo.php?script=sci arttext\&pid=S010412902011000300016\&ln $\mathrm{g}=\mathrm{en \& nrm}=\mathrm{iso}$

BILIR, N. Reduction of Adult Smoking by using Comprehensive Approach. Slides em formato ppt. [consultado em 28 jun. 2013] Disponível em: http://www.slideshare.net/stmslide/nazmi-bilir 
BLOG PROMOÇÃO DA SAÚDE. Objeto de aprendizagem sobre as temáticas que envolvem a promoção da saúde. [consultado em 27 jun. 2013] Disponível em: http://blogdapromocao-saudecoletiva.blogspot.com.br

BOYTSOV, S. Progress in NCD reduction in Russian Federation. Slides em formato

DOJHOLT, B. H. Occupation health and safety - cornerstone for sustainable health at workplaces. Slides em formato ppt. [consultado em 28 jun. 2013] Disponível em: http://www.slideshare.net/stmslide/1-bitten-hh

EL SALVADOR. The delegation of El Salvador at the Eighth World Conference on Health Promotion. 2013. [consultado em 28 jun. 2013] Disponível em: http://www.phmovement.org/sites/www.phmovement.org/files/PHM\%20El\%20Salvado r\%20Propuesta\%20declaratoria\%204\%20de\%20junio\%20El\%20Salvador\%20$\% 20$ Helsinki.pdf

GOODMAN, P. Lessons from reducing air pollution, it can be done and it works! Slides em formato ppt. [consultado em 28 jun. 2013] Disponível em: http://www.slideshare.net/stmslide/patrick-goodman-dublintechnical

HEIDMANN, Ivonete T. S.; [et al.]. Promoção à saúde: trajetória histórica de suas concepções. Texto e Contexto Enfermagem, 15(2):352-358, 2006. [consultado em 27 jun. 2013] Disponível em: http://www.scielo.br/scielo.php?pid=S0104$07072006000200021 \&$ script $=$ sci arttext

JESSE, M. Estonian experience in NCD control.2013. Slides em formato ppt. [data de consulta 28 jun. 2013] Disponível em: http://www.slideshare.net/stmslide/3-marisjesse.

ORGANIZAÇÃO DAS NAÇÕES UNIDAS. Declaração do Milênio das Nações Unidas. [online] Nova York: Organização das Nações Unidas, 2000. [consultado em 15 jun. 2013] Disponível em: www.unric.org/html/portuguese/uninfo/DecdoMil.pdf

ORGANIZAÇÃO MUNDIAL DA SAÚDE. Carta de Ottawa. [consultado em 26 jun. 2013]

www.mpba.mp.br/atuacao/cidadania/gesau/legislacao/internacionais/carta ottawa.pdf

ORGANIZAÇION MUNDIAL DE LA SALUD. Acerca de la OMS. [consultado em 26 jun. 2013] Disponível em: http://www.who.int/about/es/

ppt. [consultado em 28 jun. 2013] Disponível em: http://www.slideshare.net/stmslide/sergey-boytsov

PEOPLE'S HEALTH MOVEMENT. Promoting Health for All and Social Justice in the Era of Global Capitalism. 2013. [consultado em 28 jun. 2013] Disponível em: http://www.phmovement.org/sites/www.phmovement.org/10files/PHMStatementat8GC HP-PromotingHealthforAllandSocialJusticev4 2.pdf

SCHOOL FOR HEALTH IN EUROPE. Reducing health inequalities; experiences with health promoting schools in Eastern Europe and Central Asia. Slides em formato ppt. [consultado em 28 jun. 2013] Disponível em: http://www.slideshare.net/stmslide/goofbuijs-schools-for-health

SITE "INDEX MUNDI". Desenvolvido através de dados do CIA Factbook e apresenta quadros comparativos com dados demográficos, econômicos, entre outros. [consultado em 26 jun. 2013] Disponível em: http://www.indexmundi.com 
THE HELSINQUE Statement on Health in All Policies. 2013. [consultado em 30 jun. 2013] em: http://www.healthpromotion2013.org/images/8GCHP Helsinki Statement.pdf WESTERHOLM, B. Older people a resource - not a burden. Slides em formato ppt. [consultado em 28 jun. 2013] Disponível em: http://www.slideshare.net/stmslide/barbro-westerholm WORLD HEALTH ORGANIZATION. Nairob Call to Action. 2009. [consultado em 30 jun. 2013] Disponível em: www.gesundheitsfoerderung.ch/pdf doc xls/e/GFPstaerken/Netzwerke/Nairobi-CalltoAction-Nov09.pdf 